\title{
Basics of the NLTE physics
}

Jiří Kubát

\begin{abstract}
Basic assumptions of the NLTE approximation in stellar atmospheres are summarized. The assumptions of thermodynamic equilibrium, local thermodynamic equilibrium (LTE), and non-LTE (NLTE) are compared. It is emphasized that LTE is a poor approximation if radiative transitions dominate in stellar atmospheres. The equations of kinetic equilibrium and methods of their solution are discussed.
\end{abstract}

\section{Introduction}

Let us assume that using some spectrograph and telescope we have observed a stellar spectrum, and that we have reduced it using some data reduction software. Such spectrum is ready for its analysis. If we decide to do a model atmosphere analysis of this spectrum we need to proceed in several steps using different kind of software. First, we have to calculate a model atmosphere (spatial distribution of temperature, density, and occupation numbers), which can be done using the assumption of local thermodynamic equilibrium (LTE), for example using the popular code ATLA.S 9 (Kurucz, 1993), or without this restrictive assumption allowing the atomic level population to differ from their equilibrium values (so called NLTE), which can be done using the code TLUSTY (Hubeny, 1988). With the model atmosphere calculated we then have to determine detailed emergent spectrum. This can be done using spectral synthesis codes (like SYNTHE associated with ATLAS9 or SYNSPEC associated with TLUSTY), which calculate the formal solution of the radiative transfer equation for as many frequencies as we need, so an accurate theoretical emergent stellar radiation is obtained. The final step of the analysis is comparison of the theoretical spectrum with observations, which can also be done using a computer code.

Jiří Kubát

Astronomický ústav AV ČR, Fričova 298, 25165 Ondřejov, Czech Republic, e-mail: kubatesunstel.asu.cas.cz 
It is clear that for each step of this procedure different computer codes are used. Unfortunately, some users focus mostly to the last step (comparison of observations with the theoretical spectrum) and they are satisfied when any theoretical spectrum fits observations. They do not care about details of model atmosphere calculation and associated assumptions. However, a good fit of a simplified model does not necessarily mean that the simplification is acceptable. Taking into account more accurate physics generally leads to better models. Switching from the assumption of LTE to the assumption of NLTE is such step towards better models. Particular steps in the above mentioned spectrum analysis are influenced differently if LTE or NLTE are assumed. The initial step of model atmosphere calculation strongly depends on the assumption LTE/NLTE. Spectral synthesis is dependent on the initial step, since data input to the code is more complex for the NLTE case. The comparison step is the same if we used the NLTE model or not.

In this paper we shall discuss the physical and microscopic bases of the assumption of NLTE in a greater detail.

\section{Microscopic distributions}

Solution of the stellar atmosphere problem can be considered as the determination of spatial dependence of basic macroscopic quantities (see e.g. Mihalas, 1978; Hubeny and Mihalas, 2014, and references therein). It can also be understood (following Hubený, 1976) as searching for basic microscopic distributions, namely, the momentum distribution (which is equivalent to distribution of velocities of all particles), distribution of particle internal degrees of freedom (i.e. populations of atomic excitation stages), and distribution of internal degrees of freedom of the electromagnetic field (which is the radiation field for all frequencies and directions). Several different approximations may be applied to determination of these distributions.

\subsection{Thermodynamic equilibrium}

In thermodynamic equilibrium, all these three distributions have their equilibrium values. A system in thermodynamic equilibrium experiences no changes when it is isolated. By relaxation time we understand time necessary for the system to return back to equilibrium from a perturbed state. Macroscopic changes have to be slower with respect to relaxation time.

In thermodynamic equilibrium, the radiation field has the Planck distribution and velocities of particles follow the Maxwell distribution. Individual atomic energy levels (states) are populated according to Boltzmann distribution

$$
\frac{n_{i, j}^{*}}{n_{0, j}^{*}}=\frac{g_{i, j}}{g_{0, j}} e^{-\frac{\chi_{0 i, j}}{k T}}
$$


When the equation (11) is applied to ground levels of two successive ions, it describes the ionization equilibrium (the Saha equation),

$$
\frac{n_{0, j+1}^{*}}{n_{0, j}^{*}}=\frac{2 g_{0, j+1}}{g_{0, j}} \frac{1}{n_{\mathrm{e}}}\left(\frac{2 \pi m_{\mathrm{e}} k T}{h^{2}}\right)^{\frac{3}{2}} e^{-\frac{\chi_{I, j}}{k T}} .
$$

In these equations, $T$ is temperature, $m_{\mathrm{e}}$ is the electron mass, $n_{\mathrm{e}}$ is the electron number density, $n_{i, j}^{*}$ is the equilibrium population and $g_{i, j}$ is the statistical weight of the level $i$ of the ion $j$ (similarly for $i=0$, the ground level), $\chi_{0 i, j}$ is the excitation energy of the level $i$ from the ground level, $\chi_{I, j}$ is the ionization energy of the ground state of the ion $j, h$ is the Planck constant, and $k$ is the Boltzmann constant. Expressions for these distributions as well as discussion about influence of individual processes on maintenance of equilibrium distributions are presented also in Kubát (2010), together with references to literature.

However, thermodynamic equilibrium can not be used for description of the stellar atmosphere. As it is evident from observations of stellar spectra, the stellar radiation does not obey the Planck distribution.

\subsection{Local thermodynamic equilibrium (LTE)}

To describe the stellar atmosphere, which is the transition from the dense and hot star to almost void and cold insterstellar medium, we have to introduce the possibility of depth dependent density and temperature in the stellar atmosphere. This also allows the radiation to escape from the stellar atmosphere.

To preserve the advantages of the thermodynamic equilibrium, we introduce the local thermodynamic equilibrium, where we assume Maxwell distribution of particle velocities and Saha-Boltzmann distribution of excitation and ionization states to be valid locally using local values of temperature $T$ and electron density $n_{\mathrm{e}}$. Thus the atomic energy level populations are calculated after equations (1) and (2). The radiation field is no more in equiulibrium (does not have Planck distribution). It has to be determined by solution of the radiative transfer equation, however, the assumption of local thermodynamic equilibrium allows to use the equilibrium source function, which is equal to the Planck function.

\subsection{Kinetic equilibrium (NLTE or non-LTE)}

The assumption of kinetic equilibrium (see Hubeny and Mihalas, 2014) is more general than the assumption of local thermodynamic equilibrium. In kinetic equilibrium we determine the radiation field by solution of the radiative transfer equation and we use the exact source function. Populations of individual atomic levels are calculated using equations of kinetic equilibrium (which include the non-local influence of 
radiation on level populations), and the particle velocities are assumed to be equilibrium (Maxwell distribution). This approximation is usually called non-LTE or NLTE, which looks like negation of equilibrium. This is evidently not true, since in this "non-equilibrium" there are still particle velocities in equilibrium. Even more confusing is the expansion of the ancronym LTE, which gives "non-local thermodynamic equilibrium", which is also far from the reality. More exactly, by non-LTE we understand any state that departs from LTE. Therefore, it is better to follow Hubeny and Mihalas (2014) and call this approximation kinetic equilibrium, in contrast to the commonly used (but inexact) term statistical equilibrium. Since the ancronym NLTE is commonly used for this approximation, we shall use it also in this paper.

How close the distribution of excitation states comes to the equilibrium one, depends on a balance between radiative and collisional processes. If the collisional processes dominate, then the distribution is close to the equilibrium one. On the other hand, if radiation processes dominate, then the distribution may differ from the equilibrium one significantly. In NLTE, no assumption is made about the source function, it is consistently calculated from actual opacities and emissivities.

Generally, collisional processes tend to establish equilibrium (since particles have equilibrium velocity distributions), radiative process tend to destroy it if radiation field is not in equilibrium (which is the case in stellar atmospheres).

A detailed discussion of individual processes may be found in Kubát (2010, see Table 1).

\subsection{Basic comparison of LTE and NLTE}

Both in the local thermodynamic equilibrium (LTE) and in the kinetic equilibrium (NLTE) the maxwellian (equilibrium) distribution of particle velocities is assumed. Frequent elastic collisions between particles preserve equilibrium velocity distribution. Inelastic collisions tend to destroy it, however, if the number of elastic collisions between two inelastic collisions is very large, any deviation from equilibrium caused by inelastic collision is quickly compensated and equilibrium is preserved. In other words, for equilibrium velocity distribution it is necessary that the relaxation time is much shorter that time between inelastic collisions. Since this condition is fulfilled in most cases in stellar atmospheres, we assume in the following that the particle distribution is maxwellian (equilibrium).

The basic difference between LTE and NLTE is the behavior of calculated atomic level populations. The approximation of LTE allows their relatively very simple and fast calculation using the Saha-Boltzmann distribution (Eqs. 1 and 2). The NLTE approximation takes into account the fact that the level populations are influenced by the radiation field. In this case, the populations have to be determined by the equations of kinetic equilibrium (Eqs. 3 or 4 later in this paper).

It is the balance between several types of microscopic processes, which tells us how exact (or inexact) the assumption of LTE is. The validity of the LTE approxi- 
mation is determined by a competition between collisional and radiative processes. The radiative processes tend to destroy the equilibrium distributions (if the radiation field is not in equilibrium), while the inelastic collisions tend to establish equilibrium distribution of excitation states, however, provided that the elastic collisions are even more frequent than the inelastic ones. As collisional processes we may consider processes, where more than one particle (electron, ion, but not a photon) take part. A special case is radiative recombination, which is a radiative process (radiation is emitted), but a collision of two particles (namely an ion and an electron) must happen. For a more detailed list of microscopic processes see Table 1 in Kubát (2010).

The necessary microscopic condition for the validity of LTE is the condition of detailed balance, which means that rates of all processes are balanced by rates of their reverse processes. If the equilibrium particle velocities distribution holds, then also collisional excitation and ionization processes are in detailed balance. However, the radiative processes are in detailed balance only if the radiation field is in equilibrium, i.e. if it obeys the Planck distribution. If the mean intensity of the radiation field $J(v) \neq B(v)$, we can not reach detailed balance in radiative transitions. Since in stellar atmospheres the radiation field is generally not in equilibrium, detailed balance can not be achieved if radiative transitions dominate, and, consequently, LTE is a poor approximation there. Illustrative discusssion may be found in Mihalas and Athay (1973).

\section{Equations of kinetic equilibrium}

The general form of kinetic equilibrium equations for the state (energy level) $i$ is (Mihalas, 1978, Eq. 5-48, see also Hubeny and Mihalas 2014)

$$
\frac{\partial n_{i}}{\partial t}+\nabla \cdot\left(n_{i} \mathbf{v}\right)=\sum_{\substack{l=1 \\ l \neq i}}^{L}\left(n_{l} P_{l i}-n_{i} P_{i l}\right)
$$

$(i=1, \ldots, L)$, where $L$ is the total number of energy levels considered, and $n_{i} P_{i l}$ is the number of transitions per time unit from the state $i$ to the state $l$ (which includes both line and continuum transitions). The quantity $P_{i l}$ is the transition rate 1 . It can be expressed as a sum, $P_{i l}=R_{i l}+C_{i l}$, where $R_{i l}$ is the rate of the transition caused by absorption or emission of radiation, and $C_{i l}$ is the rate of the transition caused by a collision with a neighbouring particle, mostly with an electron. While the collisional rates depend only on local values of electron density $n_{\mathrm{e}}$ and temperature $T$, the radiative rates depend also on the radiation field, which is non-local. Detailed expressions for both collisional and radiative rates are listed in another part of this book (Bergemann, 2013). Multiplying the equation (3) by mass of the correspond-

\footnotetext{
${ }^{1}$ Note that sometimes $n_{i} P_{i l}$ is called the rate. Here we prefer to use the notation consistent with the textbook of Hubeny and Mihalas (2014).
} 
ing particle and summing all such equations over all energy levels of all species we obtain the continuity equation $\partial \rho / \partial t+\nabla \cdot(\rho \mathbf{v})=0$.

For the case of a static atmosphere $(\mathbf{v}=0)$ in a steady state $(\partial / \partial t \rightarrow 0)$, we may neglect both the time derivative and the advective term in the equation (3) and we obtain the time independent set of the kinetic equilibrium equations for static medium,

$$
\sum_{\substack{l=1 \\ l \neq i}}^{L}\left(n_{l} P_{l i}-n_{i} P_{i l}\right)=0 .
$$

However, this equation is commonly used not only for static atmospheres, but the advective term is very often neglected also for expanding stellar envelopes.

\subsection{Equilibrium level populations}

If we want to measure how much the population numbers differ from their LTE values, we use the departure coefficients ( $b$-factors) $b_{i, j}=n_{i, j} / n_{i, j}^{*}$ (Menzel, 1937), which relate actual $\left(n_{i, j}\right)$ and equilibrium $\left(n_{i, j}^{*}\right)$ populations of the level $i$ of the ion $j$.

There are two ways how to define the equilibrium populations. The first one is the natural one. The equilibrium populations in the whole stellar atmosphere are calculated using Saha-Boltzmann distributions using temperature and density distributions obtained also for the assumption of local thermodynamic equilibrium.

On the other hand, Mihalas (1978) uses the quantity $n_{i, j}^{*}$ in a different meaning. The LTE populations are calculated with respect to the population of the ground level of the next higher ion using the equation (5-14) in Mihalas (1978), which follows from Eqs. (1) and (2),

$$
n_{i, j}^{*}=n_{0, j+1} n_{\mathrm{e}} \frac{g_{i j}}{g_{0, j+1}} \frac{1}{2}\left(\frac{h^{2}}{2 \pi m k T}\right)^{\frac{3}{2}} e^{-\frac{\chi_{I j}-\chi_{i j}}{k T}} .
$$

Here $n_{0, j+1}$ is the actual population of the ground level of the next higher ion, which does not need to be an LTE value. This definition of the LTE value of population numbers reflects the fact that the radiative recombination is an equilibrium process, which causes that the ionization transitions from the highest levels of each ion $j$ are close to detailed balance.

\subsection{System of kinetic equilibrium equations}

For each atom, the kinetic equilibrium equations (4) can be written as 


$$
n_{i} \sum_{\substack{l=1 \\ l \neq i}}^{L}\left(R_{i l}+C_{i l}\right)-\sum_{\substack{l=1 \\ l \neq i}}^{L} n_{l}\left(R_{l i}+C_{l i}\right)=0 .
$$

where $i=1, \ldots, L$ and $L$ is the total numbers of atomic energy levels considered. For each atom, the system of equations is linearly dependent. We have to replace one of the equations by a 'closing equation', which sets the total atom particle density and thus makes the system linearly independent. For model atmosphere calculations we can use the charge conservation equation, which compares the total charge of all ions included in kinetic equilibrium with the charge of electrons. Alternatively, the particle conservation equation may be used. This equation uses the total number density and thus fixes the total number of particles involved. If more atoms are included in the equations of kinetic equilibrium, additional abundance equation is used as a closing equation. Kinetic equilibrium equations for each independent atom have to be closed by one such equation. These equations are listed in Kubát (2010).

The full set of kinetic equilibrium equations can be formally written as

$$
\mathscr{A} \cdot \mathbf{n}=\mathscr{B},
$$

where $\mathbf{n}=\left(n_{1}, \ldots, n_{L}\right)$ is a vector of all populations, the matrix $\mathscr{A}$ contains all rates included (it is called the rate matrix) and the right hand side vector $\mathscr{B}$ is 0 except rows with conservation laws.

\subsection{Solution of the system of kinetic equilibrium equations}

For given $\mathscr{A}$ and $\mathscr{B},(7)$ is a set of linear equations with a straightforward solution. However, the rate matrix $\mathscr{A}$ depends on the radiation field. As a consequence, we have to solve the set of equations of kinetic equilibrium simultaneously with the radiative transfer equation for all frequencies condsidered. This is a nonlinear system of equations. There is a simple iteration scheme, which consists of subsequent solution of the radiative transfer equation for given opacity and emissivity (the formal solution of the radiative transfer equation) and solution of the equations of kinetic equilibrium for given radiation field. However, this iteration scheme, known as the $\Lambda$-iteration, does not work for stellar atmospheres. More precisely, it converges so slowly that it is unusable for media which are optically thick at some frequency. Illustrative examples can be found in Auer (1984). Consequently, different iteration schemes must be used. It may be either the multidimensional Newton-Raphson method (introduced to modeling of stellar atmospheres by Auer and Mihalas, 1969, and usually referred to as the complete linearization method) or the accelerated $\Lambda$ iteration technique (introduced to radiative transfer by Cannon, 1973a b), which differs from the ordinary lambda iteration by using the approximate lambda operator. This operator allows simultaneous solution of the equations of kinetic equilibrium together with the approximate radiative transfer equation with a subsequent exact solution of the radiative transfer as a correction term. Useful reviews of accelerated 
lambda iteration methods were published by Hubeny (1992, 2003). These solution methods may be used also for the generalized problem of calculation of full NLTE model atmosphere, where additional equations of radiative and hydrostatic equilibrium are simultaneously solved.

\section{Summary}

The ancronym NLTE (or non-LTE) denotes deviation from LTE which in stellar atmospheres means that:

- the radiation field is not in thermodynamic equilibrium, its intensity has to be determined from the radiative transfer equation,

- excitation and ionization state of matter is not in thermodynamic equilibrium, the level populations have to be determined from equations of kinetic equilibrium,

- equations of radiative transfer and kinetic equilibrium have to be solved simultaneously because they are coupled,

- particle velocities are in thermodynamic equilibrium, they obey Maxwell distribution.

This approximation gives significantly better and more exact description of reality than the "standard" approximation of LTE.

Acknowledgements The author would like to thank Dr. Ewa Niemczura for inviting him to the Spring School and he would also like to apologize her for the delay in delivering manuscripts. $\mathrm{He}$ is also grateful to both referees (Ivan Hubeny and the anonymous one) for their invaluable comments. This work was partly supported by the project 13-10589S of the Grant Agency of the Czech Republic (GA ČR).

\section{References}

Auer LH (1984) Difference equations and linearization methods for radiative transfer. In: Kalkofen W (ed) Methods in Radiative Transfer, Cambridge University Press, Cambridge, pp 237-279

Auer LH, Mihalas D (1969) Non-LTE model atmospheres. III. A complete-linearization method. Astrophys. J.158:641-655, DOI 10.1086/150226

Bergemann M (2013) Spring school of spectroscopic data analyses. Springer Verlag, Berlin, GeoPlanet: Earth and Planetary Sciences, these proceedings

Cannon CJ (1973a) Angular quadrature perturbations in radiative transfer theory. J. Quant. Spectrosc. Radiat. Transfer13:627-633, DOI 10.1016/0022-4073(73)90021-6

Cannon CJ (1973b) Frequency-quadrature perturbations in radiative-transfer theory. Astrophys. J.185:621-630, DOI 10.1086/152442

Hubený I (1976) Problematika teoretického popisu hvězdných atmosfér. PhD thesis, Astronomický ústav ČSAV Ondřejov

Hubeny I (1988) A computer program for calculating non-LTE model stellar atmospheres. Computer Physics Communications 52:103-132, DOI 10.1016/0010-4655(88)90177-4 
Hubeny I (1992) Accelerated lambda iteration (review). In: Heber U, Jeffery CS (eds) The Atmospheres of Early-Type Stars, Lecture Notes in Physics, Berlin Springer Verlag, vol 401, p 377

Hubeny I (2003) Accelerated lambda iteration: An overview. In: Hubeny I, Mihalas D, Werner K (eds) Stellar Atmosphere Modeling, Astronomical Society of the Pacific Conference Series, vol 288, p 17

Hubeny I, Mihalas D (2014) Theory of Stellar Atmospheres. Princeton University Press, Princeton and Oxford, in press

Kubát J (2010) Statistical equilibrium equations for trace elements in stellar atmospheres. In: Monier R, Smalley B, Wahlgren G, Stee P (eds) Non-LTE Line Formation for Trace Elements in Stellar Atmospheres, EDP Sciences, EAS Publications Series, vol 43, pp 43-54, DOI 10.1051/eas/1043004

Kurucz R (1993) ATLAS9 stellar atmosphere programs and $2 \mathrm{~km} / \mathrm{s}$ grid. In: Kurucz CD-ROM, vol 13, Smithsonian Astrophysical Observatory, Cambridge, Mass.

Menzel DH (1937) Physical processes in gaseous nebulae I. Absorption and emission of radiation. Astrophys. J.85:330, DOI 10.1086/143827

Mihalas D (1978) Stellar atmospheres, 2nd edn. W. H. Freeman \& Co., San Francisco

Mihalas D, Athay RG (1973) The effects of departures from LTE in stellar spectra. Ann. Rev. Astron. Astrophys.11:187, DOI 10.1146/annurev.aa.11.090173.001155 